\title{
Le multipartisme démocratique au Congo est une valeur démocratique ou un désordre politique?
}

\author{
Par MFUAMBA MULUMBA Isidore* et KALALA ILUNGA Matthiesen**
}

\section{RESUME}

Il arrive de fois que nous nous demandons : si la République Démocratique du Congo est prête pour la démocratie et si elle a compris bien la démocratie. Bien que le multipartisme soit un principe (indispensable) parmi tant d'autres de la démocratie et que la démocratie pluraliste (modèle choisi par la RDC) se fonde sur la reconnaissance de la légitimité d'une pluralité effective de partis politiques, mais le parti politique n'est pas une société commerciale que tout le monde peut créer en vertu du libéralisme democraticoéconomique et dont le fonds de commerce est le désordre politique et violations droits fondamentaux en vue de conquérir du pouvoir. Etant donné qu'un tel désordre à l'impact négatif d'abord au développement de l'Etat, en suite, à la consolidation d'un Etat de droit et enfin, au respect des droits humains; il faut que le Droit encadre le phénomène de la prolifération des partis politiques en RDC. Cela étant besoin que nécessite sa diversité ethnico-tribale. Comme l'a si bien dit Goethe, "Vaut mieux une injustice qu'un désordre ». Cette pensée fait de l'ordre l'objectif sans concurrent du Droit, de la sorte, les congolais doivent faire un bon usage des principes démocratiques pour le bien être de la République Démocratique du Congo. Car la démocratie mal comprise ou abusivement utilisée risquerait de mettre le pays dans un dilemme de développement.

\section{INTRODUCTION}

Peut-être Jacques Chirac Président français déjà en son époque avait raison lorsqu'il disait «L'Afrique ne serait pas prête pour la démocratie! ». Cela pourrait être aussi dans une certaine mesure évident car, la démocratie a beau exister depuis la Grèce Antique [mais] peu de gens en saisissent les fondements et les limites ${ }^{1}$. Surtout lorsqu'on l'observe à l'africaine, d'aucuns considèrent les Etats africains comme «les mauvais élèves à l'école de la démocratie $^{2} \gg$. Cette médiocrité ne pas seulement pour le non-respect des principes qui font

* Licencié en Droit public de l'Université de Lubumbashi et Chercheur. Courriel : mfuambaisidore@gmail.com.

** Professeur Ordinaire à l'Université de Lubumbashi.

1 Yvan Dutil, Pour une meilleure démocratie Notions d'ingénierie électorale, Rapport présenté à la Commission des Institutions de l'Assemblée Nationale du Québec et au Comité directeur des États généraux sur la gouvernance démocratique, Novembre 2002, p. 2.

2 Biléou Sakpane-Gbati, «La democratie à l'africaine », in ethique publique, vol.13, n², 2011. https:/ /journals/openedition.org/ethiquepublique/679. 
le fondement de la démocratie, elle est aussi dite à un mauvais usage de ces principes. De la sorte, il nous semble que la démocratie est la plus merveilleuse des régimes pour les Etats qui respectent ses valeurs (dire mieux ses principes fondamentaux). Mais si l'on tourne la face de la médaille, elle est un régime litigieux et dangereux si on ne respecte pas ces valeurs fondamentales et/ou si l'on en fait un mauvais usage.

Il existe tout un arsenal des principes fondamentaux de la Démocratie, en l'occurrence de : Libertés fondamentales et Droits fondamentaux, Elections, Etat de droit, Séparation des pouvoirs, Le Parlement, le Pluralisme et/ou le Multipartisme démocratique, Gouvernement et Opposition, Opinion publique et liberté des médias ${ }^{3}$.

Tous ces principes assurent la bonne gouvernance d'un Etat démocratique au vrai sens du terme. Cela suppose donc, la problématique d'un bon usage. C'est-à-dire même si un Etat est démocratique, l'usage abusif de ces principes le conduira dans des crises politiques énormes et métrera en péril les droits et libertés fondamentales. Dans ce moment-là, au lieu que la démocratie soit constructive, par contre elle ne serait-ce que destructive.

Dans le contexte congolais, non seulement que le pluralisme politique fait partie des valeurs verrouillées ou intangibilisées par l'article 220 de la Constitution du 18 février 2006, en plus, la législation qui en définit les règles de jeu a voulu faire de la liberté "la norme » et de la réglementation l'exception» en matière d'association ${ }^{4}$. Oubliant que trop de liberté tue la liberté ${ }^{5}$, nous voilà aujourd'hui dans une prolifération des partis politiques jamais vu dans le monde; lesquels au lieu qu'ils soient, le fondement "des droits politiques et des libertés fondamentales qui constitue l'une des pierres angulaires de la démocratie moderne» ${ }^{6}$ tel que souhaité par le peuple congolais, deviennent plutôt un centre de leur violation.

Cependant, la présente réflexion cherche à démontrer systématiquement et méthodiquement, le pourquoi de la cacophonie politique congolaise et différentes violations des droits fondamentaux de citoyens créées par l'utilisation abusive du principe démocratique de Multipartisme. Autrement dit, nous voulons, décourager dans cette œuvre la prolifération des partis politiques (en République Démocratique du Congo), car elle est antagonique à la consolidation de la démocratie elle-même pour ce pays et elle génère d'énormes conflits tribaux et par ricochet entraine les violations des droits de citoyens. Et dans une certaine mesure cela entraine un dilemme de développement de l'Etat.

3 Cette liste n'étant pas exhaustive, mais elle contient les principaux principes indicateurs d'un Etat démocratique.

4 Voy Exposé des motifs de la loi n 04/002 du 15 mars 2004 portant organisation et fonctionnement des partis politiques.

5 Il sied de noter le but ultime qu'avait poursuivi les représentants du peuple congolais, qui était de faciliter l'exercice (des droits politiques et des libertés fondamentales) que de le compliquer par trop de réglementation et de rigidités qui finalement tuent la liberté et inhibent les talents. (Exposé des motifs de la loi $\mathrm{n}^{\circ}$ 04/002 du 15 mars 2004 portant organisation et fonctionnement des partis politiques). C'est cette facilité qui par après a constitué une porte ouverte à la prolifération des parties politiques qualitativement sans intérêt et de troubles inutiles hostiles aux droits humains.

6 Ibidem. 
Pour y arriver, nous allons faire une théorisation des concepts principaux généralités (A) avant de démonter l'utilisation abusive du principe de multipartisme en RDC et son impact sur la bonne marche de l'Etat (B).

\section{A. GENERALITES SUR LE MULTIPARTISME}

Le multipartisme est un principe majeur de la démocratie. Sans elle, plusieurs autres trouveront la difficulté de leur existence. Il en est ainsi par exemple des Elections démocratiques, opposition politique, opinion publique et liberté des médians. Mais, il serait indispensable d'en savoir le contenu (I) et formuler certaines des différentes théories développées au tour du concept parti politique, en rapport notamment de sa notion, son origine, son utilité et son danger (II).

\section{L'ANALYSE CONCEPTUELLE}

1. Le multipartisme démocratique

Le terme multipartisme, sa définition devient un peu lourde d'autant plus qu'il se présente sous plusieurs formes, peut être parfait, à parti dominant, à parti ultra-dominant et le bipartisme ${ }^{7}$.

Il nous faudrait procéder de son étymologie pour le définir aisément. Le multipartisme est un mot composé de préfixe multi du latin multus qui veut dire (nombreux, abondant ou plusieurs) et suffixe parti du latin pars (qui veut dire partie, part ou portion).

Il ressort de cette étymologie que, le multipartisme est une politique qui admet plus d'un parti politique. Il s'oppose ainsi à la politique du parti-Etat. Et dans cette logique par synonymie, on peut le designer par « pluripartisme » ou « pluralisme ${ }^{8} »$ qui parfois, s'accompagne de l'adjectif politique, ou démocratique.

Notons que le pluralisme politique est un système qui admet l'existence et l'exercice de la libre expression de différentes idées politiques, en particulier la reconnaissance des partis politiques concurrentiels 9 .

On veut parler par ailleurs de l'existence de deux (bipartisme) ou plusieurs (multipartisme) partis politiques ${ }^{10}$ qui œuvrent dans un système politique donné. Comme on le verra,

7 Voy MULUMBATI NGASHA Adrien, Introduction à la Science Politique, Lubumbashi, Edition africa, 1977, P. 119.

8 Le concept utilisé par le constituant de la RDC est le pluralisme politique (art.6).Il faut signaler que le pluralisme renvoie à la diversité, à la liberté de choix et peut prendre plusieurs figures, notamment politique, associative, religieuse, etc). Voy. Babacar Gueye, « La démocratie en Afrique : succès et résistances ", Pouvoirs $/ 2\left(n^{\circ} 129\right)$, p.5 - 26, spec. p. 7.

9 Voy Michael Walzer, pluralisme et démocratie, éditions esprit, 1997, p. 60 cité par Babacar Gueye, art cit. p. 8.

10 Le concept parti politique a une définition légale. Aux termes de l'article 2 de de la loi n ${ }^{\circ} 04 / 002$ du 15 mars 2004 portant organisation et fonctionnement des partis politiques, on enttand en RDC 
partir de deux partis politiques, dont l'un qui gouverne, et l'autre dans l'opposition, on est déjà dans un multipartisme. D'où il faut exclure l'idée selon laquelle, il y a multipartisme seulement si l'on est dans un Etat qui réunit 10 ou plus des partis politiques.

Quoi qu'il en soit, le multipartisme est favorisé par quatre facteurs : d'abord les facteurs socioéconomiques (divergences des intérêts économiques); facteurs idéologiques et religieux (la divergence des intérêts idéologiques et religieux); facteurs historiques et nationaux (la divergence de particularismes historiques et sociales qui en divisant les différents éléments de la population) et enfin, les facteurs institutionnels (système électoral d'un pays, par exemple la représentation proportionnelle favorise les multipartisme intégral, scrutin majoritaire à deux tours favorise multipartisme et à un tour favorise le bipartisme $)^{11}$.

Par opposition on parle de monopartisme qui souvent se trouve dans les Etats dictatoriaux dans lesquels il n'existe aucune liberté politique. Le monopartisme est né en Allemagne au début du 20eme siècle et en Italie et par la suite s'est implanté en U.R.S.S. et dans les pays de la démocratie populaire d'Amérique latine, d'Asie et d'Afrique ${ }^{12}$. Le Congo en a vécu aussi une expérience dans son histoire avec Zaïre. Il s'agit du parti-Etat $M P R$ sous régime de Président MOBUTU SESESEKO KUKUBENDO WA ZABANGA.

Pour expliquer mieux ce concept, il s'agit d'une valeur ou d'un principe démocratique qui exige la présence des deux ou plusieurs partis politiques dans un Etat démocratique, pour permettre l'égalité de chances d'être élu et aussi permettre à ce que le peuple exprime son scrutin c'est-à-dire donner mandat public aux personnes voulues.

\section{2. valeur démocratique}

Le terme valeur peut avoir plusieurs interprétations selon le contexte, mais en tenant compte de notre sphère de raisonnement, la valeur est une importance accordée à une chose $^{13}$. Et cette chose dans le cas sous examen c'est la démocratie. Cette dernière selon son étymologie entendue comme un «gouvernement par le peuple ${ }^{14} »$.

d'un parti politique « une association des personnes physiques de nationalité congolaise qui partagent la même idéologie et le même projet de société, en vue de conquérir et d'exercer démocratiquement et pacifiquement le pouvoir d'Etat».

11 Voy Roger-Schwartzenberg, sociologie politique, Paris, Ed. Montchrestien, 1974, P. 478-484.

12 MULUMBATI NGASHA A., op cit, P. 130.

13 LAROUSSE, Dictionnaire de Français, ed. 2006, P.40.

14 Plus concrètement, régime dans lequel tous les citoyens possèdent de manière égalitaire un droit de participation (vote) et un droit de contestation à l'égard du pouvoir (liberté d'opposition). Cependant la liberté d'opposition n'est reconnue que dans la démocratie libérale, mais non dans la démocratie populaire. Voir Serge GUINCHARD et Thierry DEBARD, Lexique des termes juridiques, Éditions Dalloz, Paris, 2018, p. 647. 


\section{3. désordre politique}

Le désordre est une agitation politique ou sociale. ${ }^{15}$ Et aussi ce terme, nous le comprenons dans le sens de manque de discipline dans la manière d'entreprendre une activité quelconque.

Il convient qu'on note d'ores et déjà le désordre est antinomique au Droit. Ce sont les deux ennemis jurés. Mais la politique a besoin de Droit pour être régulée et équilibrée de peur que l'homme n'exploite l'homme ${ }^{16}$.

Ainsi, l'ordre fait quitter la politique de l'état sauvage à l'état de Droit. Et on peut aller plus loin pour dire que l'injustice peut être admissible par le Droit ${ }^{17}$ tout comme en politique mais jamais le désordre pour un Etat de droit digne de ce nom. En plus, dans l'échelle des valeurs l'ordre prédomine car la justice elle-même fait partie intégrante de l'ordre; d'où sauvegarder ce dernier serait sans doute sauvegarder la première.

\section{NOTION, ORIGINE, UTILITE ET DANGER DE PARTI POLITIQUE}

Dans une démocratie, et plus précisément dans le domaine politique, le multipartisme implique qu'un grand nombre de groupements et d'associations librement formés se retrouvent réciproquement en situation de concurrence pour gagner de l'influence sur la vie sociale et politique. Ces groupements peuvent être de nature politique, économique, religieuse, ethnique ou autre ${ }^{18}$.

Il va d'une utilité grandiose de définir par ailleurs, le concept parti politique l'âme du mot multipartisme.

Le terme regorge plusieurs définitions que nous ne pouvons tout donner. Mais, soulignons avec Antony Downs, " un parti politique, c'est une coalition d'hommes qui cherchent à prendre le contrôle, par moyens légaux de l'appareil du pouvoir ${ }^{19}$.

15 Idem, p.119.

16 La politique dans sa conception traditionnelle, l'on peut user de tous les moyens même illégaux pour atteindre l'objectif, notamment la conquête du pouvoir, son exercice et sa conservation plus longtemps que possible.

17 GOETHE disait : "je préfère mieux une injustice qu'un désordre ». Sa pensée fut développer en principe selon lequel «Mieux vaut une injustice qu'un désordre ». Il veut montrer que le Droit est avant tout à la recherche de l'ordre ». Etienne ILUNGA KABULULU, Introduction géneral a l'étude du Droit, A l'intention des Cadres Administratifs de l'IG/PNC, RDC, JANVIER 2012, p. 11.

18 PAULA BECKER ET JEAN-AIME A., Qu'est-ce que la démocratie?, Auteurs (texte original en allemand) : Paula Becker Traduction en français: Rabary-AndriamandayVoahanitriniaina Coordination : Jean-Aimé A. Raveloson Antananarivo, septembre 2008, p.13.

19 A. DOWENS, An Economie Theory of Democracy, New York, Harper, 1957, P.24 (traduit par jean et Monica Charlot). 
Les origines d'un parti politique sont diverses que selon MAURICE DUVERGER, ${ }^{20}$ le regroupe en deux grandes catégories (d'une part, les partis politiques d'origine parlementaire et électorale et d'autre part, les partis politiques d'origine extérieure au parlement).

Nous soulignons avec PAULLA que, malgré les critiques que l'on peut adresser aux partis politiques en ce qui concerne les résultats de leur travail, il faut reconnaître qu'ils constituent malgré tout un élément nécessaire et indispensable à toute démocratie. Le peuple peut exercer sa souveraineté par le biais de ces partis politiques; c'est vraiment grâce à eux qu'il peut agir de manière effective ${ }^{21}$.

En tout état de cause, dans un Etat démocratique, les partis politiques peuvent se faire en bloc majoritaires et oppositionnels en vertu du " principe du Gouvernement et Opposition ». Selon ce principe, dans un Etat démocratique, il y a toujours une opposition qui manifeste ses idées parallèlement au gouvernement.

\section{B. L'UTILISATION ABUSIVE DU PRINCIPE EN RDC ET SON IMPACT SUR LA BONNE MARCHE DE L'ETAT.}

La démocratie pluraliste est fondée sur la reconnaissance de la légitimité d'une pluralité effective de partis politiques, et de leur alternance au pouvoir. Cette conception de la démocratie a représenté l'une des différences essentielles entre les démocraties libérales « occidentales » et les démocraties populaires (et l'Union soviétique) ${ }^{22}$.

Comme nous l'avons vue, le multipartisme est un principe parmi tant d'autres de la démocratie. C'est une valeur démocratie à grande échelle dans l'émergence et consolidation d'un Etat démocratique.

Il est l'une des conditions essentielles de la démocratie libérale, laquelle suppose que le peuple choisisse ses gouvernants. Et pour faire le choix, on a besoin des plusieurs possibilités, plusieurs combinaisons ${ }^{23}$.

La réalisation de ces finalités, n'est possible que lorsqu'on en fait le «bon usage ». Au cas contraire, c'est les crises; violations des droits fondamentaux; création des mouvements rebelles et les guerres civiles etc. Tous ceux-ci recule l'Etat en arrière au lieu qu'il progresse.

Notre constat sur l'utilisation abusive du principe de pluripartisme en République Démocratique du Congo soulève la problématique du nombre des parties politiques (I). Le

20 MAURICE DUVERGER, Partis politiques, Paris, Edition A. Colin, 1968, P. 34.

21 PAULA BECKER ET JEAN-AIME A., op cit, P.13.

22 La démocratie populaire est un régime politique marxiste et totalitaire institué au lendemain de la Seconde Guerre mondiale dans les États d'Europe centrale et orientale situés dans la zone d'influence de l'URSS. Ces régimes ont été créés sur le modèle soviétique, avec cependant des éléments originaux plus ou moins marqués. Les événements survenus dans la plupart de ces États fin 1989 ont conduit à la fin de ce régime politique et à la mise en place (malgré certaines difficultés) d'une démocratie pluraliste. Voy. Thierry DEBARD, op cit, p. 648.

23 Babacar Gueye, art cit. p. 7. 
contenu de cette problématique et les sous questions qu'elle génère, nous permettrons de faire des observations et suggérer pour l'amélioration dans le proche avenir (II).

\section{PROBLEMATIQUE DU NOMBRE DES PARTIS POLITIQUE EN RDC.}

La République Démocratique du Congo a choisie comme modèle, la démocratie pluraliste. Sa Constitution réserve l'économie des articles premier à huitième à cette matière. Et l'article 6 affirme ouvertement la reconnaissance du pluralisme en République Démocratique du Congo (al.1) et fait obligation le respect des principes de démocratie pluraliste, d'unité et de souveraineté nationales (al.4). En s'inspirant de l'histoire qu'a connue le pays sous la deuxième République, qui dirait : «le fait créent les lois », le constituant du 18 février 2006 a consacré le principe du «pluralisme politique » et a érigé en une infraction imprescriptible de haute trahison "l'institution d'un parti unique ${ }^{24}$ ».

Comme si cela ne suffisait pas, l'article 8 de la Constitution sacralise même les droits liés à l'existence de l'opposition, à ses activités et à sa lutte pour la conquête démocratique du pouvoir. Et aussi le pluralisme politique et syndical est intangibilisée ${ }^{25}$ par l'article 220.

Nonobstant ce qui précède, les partis d'Opposition congolaise (tout comme de la majorité) doivent faire attention dans l'exercice de ses droits, la constitution ne garantit pas seulement les droits mais aussi elle impose les obligations ${ }^{26}$. Même si ceci est la chose la

24 Article 6 alinéa $1^{\text {er }}$ et article 7 alinéa $2^{\text {ème }}$, de la Loi n ${ }^{\circ} 11 / 002$ du 20 janvier 2011 portant révision de certains articles de la Constitution de la République Démocratique du Congo, Journal Officiel Numéro spécial 5 février 2011.

25 La technique de rendre certaines dispositions intangibilité est ainsi rependu dans le constitutionnalisme africain avec raison, pour ainsi « placer certains droits hors d'atteinte du pouvoir constituant dérivé » (V. SAINT-JAMES, " Hiérarchie et conciliation des droits de l'homme », J. ANDRIANTSIMBAZOVINA et alii (dir.), Dictionnaire des droits de l'homme, Paris, Puf, 2008, pp. 477-481, spéc. p. 477) et empêcher toute modification avec intention de réduire leur jouissance. La constitution de la République Démocratique du Congo a regrouper dans cette catégorie « la forme républicaine de l'Etat, le principe du suffrage universel, la forme représentative du Gouvernement, le nombre et la durée des mandats du Président de la République, l'indépendance du pouvoir judiciaire, le pluralisme politique et syndical, les droits et libertés de la personne, ainsi que les prérogatives des provinces et des entités territoriales décentralisées».

26 Le titre deuxième de la loi n ${ }^{\circ} 07 / 008$ du 04 décembre 2007 portant statut de l'opposition politique, respectivement consacré aux droits et devoirs de l'Opposition politique donne une liste imposante des devoirs que doivent remplir les partis politiques et groupement de l'Opposition. L'article 16 les résume de la sorte : « respecter la Constitution, les Lois de la République et les Institutions légalement établies; défendre les intérêts supérieurs de la Nation; s'abstenir de recourir à la violence comme mode d'expression et d'accès au pouvoir; privilégier le dialogue et la concertation sur les grandes questions d'intérêt national et dans la résolution des différends politiques; promouvoir le pluralisme politique et reconnaître le droit de la Majorité à gouverner; promouvoir la culture démocratique notamment par la tolérance, la non-violence et le soutien du principe de l'alternance dans le cadre d'une lutte politique pacifique; concourir, par la libre expression, à la formation de l'opinion publique et former et informer ses militants sur les questions touchant à la vie nationale ». 
plus ignorée par les uns et beaucoup plus oubliées par les opposants moins scientifiques ${ }^{27}$. Comme on le dit "les mêmes causes produisent les mêmes effets », si les élus délégués par les partis pour qu'ils soient mandataires du peuple(le mandant), le niveau d'instruction ne convient, une fois devant les questions plus spéciales, s’ils ne se taisent pas, comme "la colère est l'argument de faible», ils vont s'énerver et créer le désordre politique, sans tenir compte de conséquence que les mandants peuvent subir.

L'opposition ne veut pas non plus dire un désordre, d'autant plus que la majorité et l'opposition toutes poursuivent l'intérêt supérieur de la population (souverain primaire). Chose étonnante, est de voir les militants sur les questions touchant à la vie nationale ne font aucun bruit, mais la question touchant le leader d'un parti politique ou un fidèle cardage de leur parti (pourtant responsable de fait lui reproché) ils sèment les troubles.

Nous insistons sur ce point du faite que suite aux désordres politiques, la RDC et sa population sont victimes des beaucoup de pertes de biens publiques voire violations graves des droits de l'homme (notamment les pertes des vies humaines), alors que consacrés (droits) par les instruments internationaux et la Constitution de la RDC.

Un constat amer est d'entendre de propos comme " il n'y a pas de révolution sans le sang $"{ }^{28}$ Cette philosophie est erronée selon nous, car s'il n'y avait pas de révolution sans le sang, les leaders pouvaient prendre leurs parents et alliés pour mourir avec eux. Mais pour la plupart, nombreux de leurs proches ne vivent même pas dans le sol congolais le temps qu'ils préfèrent révolutionner les autres ${ }^{29}$.

Si jacques Chirac était parvenu à dire que : "l'Afrique n'est pas encore près pour la démocratie ", il pouvait à ce qui nous concerne avoir raison du point de vue factuel. La démocratie, est un régime merveilleux pour ceux qui respectent ces principes fondamentaux. Elle peut de fois ressembler à un cauchemar pour les utilisateurs abusifs de ses prin-

27 Cela est d'ailleurs prouvé par l'analyse faite par professeur YAV KATSHUNG en voyant l'incompétence notoire dans certains élus (produits de partis politiques sans éthique qui délèguent n'importe qui en vue de lui donner le pouvoir pendant qu'ils ne connaissent pas que le pouvoir c'est un mandat).

Professeur dit : « En outre, l'élection ne doit pas être une marchandise, objet de commerce où, on se fait élire pour passer d'autres clients (ses suppléants) et qui dans un cas comme un autre, ne jouent pas toujours le rôle attendu de l'élu et parfois, vont à contre-courant des promesses de l'élu lui-même ».

D'où, au-delà de l'âge et du casier judiciaire supposé vierge (nos institutions judiciaires ne disposent même pas d'archives), la Loi électorale devra être amendé ou modifié en introduisant aussi le critère de niveau d'instruction (au moins diplômé d'Etat ou l'équivalent) et de « capabilité » pour être en mesure de rédiger un rapport et de s'exprimer en 2 À l'instar de l'Ouganda Français, en plus d'une langue nationale afin de porter la voix de ses électeurs. YAV KATSHNG JOSEPH, $R D C$ : «Nécessité d'un Profil Remodelé des Députés, Sénateurs, Gouverneurs, etc? », Le Contrôle Citoyen de la RDC, www.controlecitoyen.com, 18 février 2010, p 2.

28 Selon les opposants révolutionnaires, qui provoquent les émeutes et occasionnent les affrontements des civiles et les militaires.

29 Contre argument donné par les partisans de la majorité politique. 
cipes. C'est le cas du principe de pluralisme politique sous examen en RDC utilisé abusivement.

Bien évidemment, en vertu du multipartisme un Etat doit avoir plusieurs partis politiques dans son système politique, mais combien? N'est-il pas loisible de limiter les seuils? La réponse selon nous est relativisée, elle dépend du factuel d'une société à une autre par rapport à son histoire, ses problèmes et ses besoins les plus cherchés. Quid de la RDC?

Il est vrai que le législateur congolais a adopté la loi n 04/002 du 15 mars 2004 portant organisation et fonctionnement des partis politiques, en vue de mettre en œuvre la résolution $\mathrm{N}^{\circ} \mathrm{DIC} / \mathrm{CPJ} / 04$ du 18 avril 2002 du Dialogue inter-congolais ${ }^{30}$, selon les termes duquel le Gouvernement de transition a été chargé " d'assurer la libéralisation effective de la vie politique et associative sur tout le territoire national », et le Parlement de transition a été aussi chargé « d'élaborer à cet effet une nouvelle législation » ${ }^{31}$.

Pour ce législateur, la liberté d'association est une règle (norme) et la règlementation est l'exception. Ce principe pour nous, est à la base de la prolifération des partis politiques. Or, les partis politiques pour assurer les meilleurs de la démocratie pluraliste dans un Etat, doivent être qualitatifs et non quantitatifs, car aux termes de l'article 6 de la Constitution ${ }^{32}$, ils ont des fonctions à remplir.

L'histoire du Congo est vaste, mais certains points saillants doivent nous préoccuper. Le Congo à partir de son territoire présente des grands problèmes historiques relatifs au séparatisme (1), sa diversité ethnico-tribale est étonnante (2) et le besoin du développement s'impose (3). Ces problèmes avec un pluralisme politique irréfléchi, ne peuvent pas avoir des solutions efficaces.

\section{SEPARATISME}

Historiquement, le Congo a connu deux formes de territoire (forme fédérale et unitaire). Depuis les assises de Berlin la question a été traité est le consensus en était trouvée de peur que d'une part, l'on ne détruise pas ce qui a été construit difficilement par Léopold II en collectant les signatures des chefs traditionnels si fédéralisme pouvait être adopté. Et d'autre part, vu l'immensité de son territoire son développement devrait être comparable à " une dent du coq» si on pouvait adopter l'unitarisme $e^{33}$.

30 Voy La Résolution du Dialogue inter-congolais $\mathrm{N}^{\circ} \mathrm{DIC} / \mathrm{CPJ} / 04$ du 18 avril 2002 relative à la libéralisation effective et totale de la vie politique et associative en République Démocratique du Congo.

31 Exposé des motifs de la loi n 04/002 du 15 mars 2004 portant organisation et fonctionnement des partis politiques.

32 Article 6 dispose : « Les partis politiques concourent à l'expression du suffrage, au renforcement de la conscience nationale et à l'éducation civique. Ils se forment et exercent librement leurs activités dans le respect de la loi, de l'ordre public et des bonnes mœurs "».

33 Pour savoir beaucoup sur ce, lisez BANZA MALALE MAKUTA Gabriel, les aspects juridiques dans les enjeux des crises congolaises, des origines à nos jours (1860-2006), p. 839 et 840. 
Pour voir que ce danger historique est permanant, bien que la RDC a connu la forme fédérale et la forme unitaire, chaque fois que le peuple est appelé à choisir librement sa Constitution, la forme fédérale a toujours été adoptée mais terme forme fédérale n'a jamais été appliqué comme voulue. Il fut ainsi pour la loi fondamentale du 19 mai 1960; à la table ronde politique de Bruxelles du 20 janvier au 20 février 1960; de la Constitution de Luluabourg, adoptée par référendum national et promulgué le 1er août 1964 a connu également la voie fédéraliste; de la constitution de la conférence nationale souveraine (CNS) tenue à Kinshasa de 1991 à 1992; et de l'actuelle Constitution (18 Février 2006) concerne également le même procédé de gestion de l'Etat ${ }^{34}$.

Il est vrai que, s' il est évité d'user des termes " Etat fédéral " malgré la pratique du fédéralisme, sans doute, c'est par peur du séparatisme. $\|^{35}$

Même si dans certaines formes instaurées par les différentes constitutions apparaissaient les éléments d'un Etat fédéral, notamment la loi fondamentale, les mécanismes qui sont consacrés importent une organisation unitaire ${ }^{36}$. Parmi ces mécanismes ont cité la terminologie (foncièrement) unitariste : province au lieu de l'Etat fédéré; l'indivisibilité du pouvoir judiciaire; etc.

D'ailleurs, il va dans la même logique de séparatisme les deux sécessions que Congo a pu connaitre (KATANGA ET KASAI). L'indépendance de l'Etat du Congo avait conduit l'Etat en chaos, le KATANGA et le KASAI avaient déclarés la sécession, les belges avaient pris fuites et la Belgique en voyant cela envoya les troupes et l'ONU, il y avait eu guerre et après la situation a été calmée et Congo était resté uni.

Par ailleurs, le nombre des partis politiques devient de plus en plus élevé ce qui mène bien sûr dans le chemin de séparation. Car du fait que le nombre augmente, les conflits des intérêts augmentent et cela débouchera sur les conflits de séparation. Jusqu'aujourd'hui on ne cesse d'étendre les murmures de BAKATAKATANGA qui veulent que le Katanga soit un Etat de part entière et d'autres provinces encore qui veulent se jeter dans ce mouvement comme KIVU.

\section{DIVERSITES ETHNICO TRIBALES}

Chaque société génère son propre Etat dont les caractéristiques les plus ultimes ne peuvent être transposées ailleurs, de sorte qu'il n'existe pas un Etat prototype universel, mais des Etats dont les trais reflètent l'histoire, la géographie et tout ce qui contribue à identifier la culture propre à leurs peuples. Chaque Etat présente une personnalité spécifique faite de

34 NGOMA BINDA (P), La participation politique, IFEP, Kinshasa, 2005, p.59-60; THIAM (D), Le Fédéralisme africain, Présence Africaine, Paris, 1972, p.62 - 63; voy Isidore NDAYWEL è NZIEM, La constitution de la IIIème République du Congo-zaïre, adoptée par la CNS? édition en 2 volumes, tome 1, L'Harmattan, 2002, p. 2-4.

35 Etienne ILUNGA KABULULU, op cit.

36 Jacques Djoli Eseng'Ekeli, Droit constitutionnel, l'expérience congolaise (RDC), L'Harmattan, 2013, p. 81. 
particularités non transposables. Il va de l'Etat comme de l'être humain, irréductible généralement et rebelle selon les règles biologiques à tout clonage ${ }^{37}$.

De cette idée nous déduisons que tout Etat est identique à l'image de son Etat, inutile de le comparer avec les autres.

Le Congo est un Etat d'une nature jamais vue dans le monde du point de vue de sa diversité ethnique. BANZA MALALE enseigne qu'il est un Etat atypique du monde, qui a une nature juridique conventionnelle, soumis à un régime international sui generis.

Aucun n'Etat dans le monde n'est comparable à celui du Congo en rapport avec les diversités ethnico tribales. Le Congo a une population constituée d'une communauté de 450 tribus qui peuvent être réparties en cinq groupes principaux : le groupe bantou; le groupe soudanais; le groupe pygmoïde et le groupe hamite. La diversité ethnique s'accompagne d'un multiple de langues. La population parle environ 250 dialectes rattachés soit au groupe des langues soudanais soit au groupe des langues bantoues ${ }^{38}$.

Un Etat comme celui-ci pour éviter de conflits tribaux, il faut nécessairement lutter pour constituer une nation. Alors que même si le constituant qualifie le Congo d'une nation, la science prouve au contraire, que "nous ne sommes pas encore arrivé à une nation ${ }^{39}$ car le vouloir vivre ensemble nous est cher comme "l'arme de crocodile".

Les partis politiques en RDC, la statistique nous montre qu'ils concourent à ce jour au nombre plus de $400^{40}$ (le nombre proche aux tribus de cette dernière) et un spécialiste ${ }^{41}$ démontre que chaque parti a tendance d'avoir un leader politique rattaché à une tribu bien déterminée ce qui est un danger permanant ${ }^{42}$. Le nombre des partis politiques présentés aux élections de 2018 est de 599 et les regroupements politiques $77^{43}$.

Les conflits tribaux sont plus vieux que le Congo lui-même, c'était une politique malheureusement qui est restée jusqu'à ce jour ancrée dans la conscience des congolais.

37 Jacques Djoli Eseng'Ekeli, op cit, p. 63.

38 WHIKA TSHIBINDA BAUBOUIN, Cours de Droit structures et institution traditionnelles africains,G2 Droit, UNILU, 2014-2015, P77.

39 MUSAFIRI NALWANGO Paul, Cours de Droit constitutionnel : théorie générale de l'Etat, G1 Droit, UNILU, 2013-2014.

40 La liste de juillet 2006 donnait 400 partis politiques enregistrés auprès du ministre de l'intérieur. $\mathrm{https//fr.m.wikipedia.org/wiki/liste-des-partis-politiques-de-la-republique-democratique-du-cong.}$ Dernière modification le 19 juillet 2019 à $15 \mathrm{~h} 42$. Consulté le 18 septembre 2019, à $02 \mathrm{~h} 42$.

41 BANZA MALALE G., cours de Droit constitutionnel congolais, G2 Droit, UNILU, 2014-2015. Inédit.

42 Et cela est une violation de l'article 5 de la de la loi portant organisation et fonctionnement des partis politiques, qui exige qu'on veuille dans la création, organisation et fonctionnement, les partis politiques «à leur caractère national et ne peuvent ni s'identifier à une famille, à un clan, à une tribu, à une ethnie, à une province, à un sous-ensemble du pays, à une race, à une religion, à une langue, à un sexe ou à une quelconque origine, ni instituer toutes discriminations fondées sur les éléments ».

43 Cheik FITA, « voici les 599 partis politiques et 77 regroupements pour les élections RDongo de 2018 », 15 mai 2018. www.cheikfitanews.net/2018/05/voici-les-599-partis-politiques-et77-regroup ements-pour-les-elections. Consisté le 19 septembre $02 \mathrm{~h} 10$. 
Pour élire un mandataire la plupart veulent choisir le frère ou la sœur tribale même s'il n'a pas un projet de société.

Est-ce que l'on comprend c'est quoi la démocratie?

En plus, une nouvelle forme de désordre est à constater dans le dédoublement des partis politiques, sans logique formelle imposée par les textes qui régissent la matière. Ce qui a poussé certaines ONG de défenses des droits de l'homme à demander au ministre de l'intérieur (à l'époque Henri MOVA SAKANI) de prendre toutes les dispositions pratiques pour vide la question, pour ne pas retomber dans les erreurs de ses prédécesseurs qui avaient mise en place une politique de dédoublement de plusieurs partis politique de l'opposition. Une situation qui avait provoqué des tensions à la base entre différents militants de différentes structures des partis politiques ${ }^{44}$.

-Cette prolifération des partis nous semble dangereuse pour la stabilité du pouvoir, la paix sociale, le respect et promotion des droits de l'homme et la sécurité nationale. C'est évident comme on peut le constater, plusieurs avant de former les partis politiques étaient d'abord rebelles; ils ont violés, volés, tuer etc. Et une fois qu'il est appelé, moyennant un pot-de-vin il abandonne (temporairement) son mouvement, récompense ses miliciens et crée son propre parti politique grâce à la procédure libérale et simpliste de la création des partis (tel que dit supra) pour arriver aux pouvoirs. Ce qui ne l'empêche pas d'agir en héro dans l'ombre, parfois financer ses miliciens pour semer de trouble chaque fois qu'il sent ses intérêts en péril. Alors que tout parti s'est engagé à promouvoir la démocratie en son sein, les droits de l'homme et les libertés fondamentales et à ne jamais recourir à la violence ni à la contrainte comme moyen d'expression, d'action politique et d'accès ou de maintien au pouvoir ${ }^{45}$.

\section{LE BESOIN DU DEVELOPPEMENT}

Si réellement comme un adage le dit : "l'union fait la force », donc en toute logique valide «la séparation fait la faiblesse».

Le Cogo (RDC) est jusqu'à ce jour un Etat sous développé même si il est potentiellement riche du monde tel que la théorie l'enseigne. Même si les causes sont légion, la pluralité politique devient de plus en plus une autre. Si on n'en tire pas attention, sans être prophète de malheur, le développement de la RDC est difficile. ${ }^{46}$

Pour se rendre compte, certains partis au lieu de se former normalement en adoptant les conditions requises, veulent passer par des moyens illégaux, entre autres : la rebellions, guerres civils etc. Et qui conque passe par ces moyens détruit les biens publics; les vies humaines, les droits de l'homme et j'en passe. Quelle serait un tel parti même si arriverait à

44 https://www.radiookapi.net/2018/03/20/actualite/politique/rdc. Consisté le 19 septembre 03h 19.

45 Article 5 de la loi $n^{\circ}$ 04/002 du 15 mars 2004 portant organisation et fonctionnement des partis politiques.

46 Cette opinion nous engage, nous ne sommes pas lié par les sentiments patriotiques car la science se veut objective et doit être vérité, vérifiée et vérifiable. 
trouver le pouvoir politique (l'objectif sacro-saint du parti)? Cette destruction et attaque contre les biens publics retourne l'Etat dans sa case départ.

\section{OBSERVATIONS ET SUGGESTIONS}

La République Démocratique Congo à l'heure actuelle est dans un désordre politique, confondu à la valeur démocratique «multipartisme ». Arriver déjà dans les 599 partis politiques et on continue à créer d'autres, expose l'Etat. On peut se demander qui autre dans le monde peut compter le nombre des partis que la République Démocratique du Congo compte aujourd'hui?

En observant nous trouvons que les partis politiques ne font qu'augmenter en quantité mais en qualité rien du tout.

La compréhension negro africaine dudit principe n'est pas la même avec celle des autres. Pour notre étude nous trouvons que le comportement des leaders politiques congolais, ne traduit pas la démocratie.

Un Etat comme Congo selon nous, avec ses diversités tribalo-ethniques ne pouvait pas avoir un nombre excessif des partis politiques. Car il est vrai et d'une vérité absolue que les partis entre eux ne s'entendent pas à cause de divergences de leurs intérêts. Lesquels intérêts d'autres sont beaucoup plus tribaux, or comme nous l'avons soulignés ci-haut on court un danger du séparatisme.

D'ailleurs, à ce propos en prenant l'occurrence des vieux Etats protagonistes de la démocratie que nous nous qualifions les Etats pères de la démocratie, on ne trouvera pas le nombre des partis politiques excessif comme pour la RDC.

La pratique des subdivisions des partis politiques en deux (appelée dédoublement) aussi, vient inquiéter l'usage de ce principe démocratique en RDC.

La constitution de la République Démocratique du Congo, à propos de la qualité scientifique pour solliciter création d'un parti politique reste muette alors que même si aujourd'hui on peut admettre le statut légal des droits de l'homme et libertés publiques (notamment des droits civils et politiques), la constitution demeure une source par excellence. Cela aussi montre une faiblesse, car une telle disposition devrait ressortir de la constitution même sommairement, pour éviter les différentes instabilités de la loi électorale que l'on modifie à souhait.

En rapport avec ce qui vient d'être observé, nous recommandons d'imiter les Etats de l'occident qui ont montré aux africains la culture démocratique; comme qui dirait « tel père tel fils ». Les congolais doivent éviter de se faire démocrates plus que les initiateurs de la démocratie eux-mêmes. Tout ne pas à prendre en bloc. Même une reforme demande une certaine logique de principe, sans laquelle elle devient une dégradation (Doyen Adan Haddad).

Il ne pas aussi du tout mal d'innover, si nous voulons innover ou prouver au monde extérieur notre volonté de lutter contre ce que nous avons connu sous la deuxième République 
« parti-Etat ». L'innovation aussi sans adaptation est une destruction (Doyen Adan Haddad).

La vérité reste que plus de partis politiques, il y a plus des problèmes car chaque parti cherche ses intérêts égoïstes au premier plan, ceux du peuple viennent en deuxième position. Les Etats qui en ont moins, ils connaissent surement les conséquences, pendant qu'ils n'ont même pas de diversités tribalo-ethniques comme la RDC.

Ainsi donc, pour éviter que ceci devienne un désordre, le Droit devrait revoir les principes et règles mise en place pour création des partis politiques ${ }^{47}$, en mettant notamment de verrou procédural et conditionnel afin de dépister la capacité du futur parti de se conformer aux devoirs légaux. Bien que cela pourrait s'interpréter en une "injustice », mais vaut mieux ça que le désordre.

La qualité des leaders doit aussi être prise en compte dans la création des partis politiques. Le constituant consent à ce que la disposition de l'article 5, soit utilisée conjointement avec les dispositions des articles 72 (fixant les conditions qu'un candidat doit remplir pour être élu comme Président de la République), 102(fixant les condition qu'un candidat aux élections législatives) et 106 (fixant les conditions pour être candidat membre du Sénat) de la même Constitution ${ }^{48}$.

Bien qu'aucune condition relative à la capacité intellectuelle n'est requise, à la lumière de la science nous considérons que la compétence est très nécessaire, professeur YAV KATSHUNG demande même que "la Loi électorale devra être amendée ou modifiée en introduisant aussi le critère de niveau et préfère la détention d'un diplôme d'Etat au-delà de l'âge et du casier judiciaire supposé vierge $»^{49}$.

Et cela est encore prouvé par professeur MULUMBATI NGASHA dans ce termes : «... les organes qui animent le parti politique doivent être dirigés par les hommes et des femmes compétents, c'est-à-dire qui, par leur savoir-faire et leur savoir être, permettent au parti politique de gagner les élections $»^{50}$. Le savoir-faire et savoir être dont question ici, n'est pas créer les dégâts; émeutes sanglantes et autres antivaleurs, même si tous les moyens peuvent être utilisés pour atteindre l'objectif. In contrario, c'est du savoir-faire dans la légalité.

En effets, étant éclairé par la science, nous proposons que cette pratique ne peut pas émerger un Etat, car si dans un Etat tout le monde veut être commerçant qui achètera

47 Ces règles ont un caractère purement simpliste en fonction de l'une des neuf innovations apportées par la loi en vigueur, qui se force d'éviter que la formalité d'enregistrement ne se transforme en agrément et de protéger les fondateurs d'un parti politique contre les manœuvres dilatoires des autorités compétentes en matière d'enregistrement, conférant ainsi la valeur juridique de l'acte d'enregistrement au récépissé de la demande d'enregistrement et à la preuve du dépôt du recours au Greffe de la Cour Suprême de Justice contre l'arrêté de rejet de cette demande si le Ministre ayant les Affaires Intérieures dans ses attributions ou la Cour Suprême de Justice saisie d'un tel recours ne se prononce pas dans le délai légal.

48 Constitution de la République Démocratique Congo du 18 février 2006.

49 YAV KATSHUNG J., art cit.

50 MULUMBATI NGASHA A., op cit, p. 119. 
l'autres? Quel serait le niveau de concurrence? C'est pareil avec la politique au Congo, les faux politiciens ou politiques que nous qualifions comme commerçants (l'évidence), ayant comme fonds de commerce le désordre politique, à n'aucun cas ne peuvent pas émerger l'Etat du Congo. Si on peut se demander Pourquoi? Selon, les investigations des chercheurs en la matière, le Congo est un Etat consensuel ${ }^{51}$. Cette qualité qui a fait naitre le Congo, n'est pas de facto plutôt elle est de jure. Donc elle reste et elle restera attachée cette nature pour toujours, car la puissance d'un Etat réside dans sa nature. Or, en réalité ladite nature semble être supprimée parce que les considérations politiques et tribales l'ont étouffées depuis lors, jusqu'à ce jour.

Le pouvoir public (entendons le Parlement) a cependant, la responsabilité de disposer par les règles générales les conditions des sévères et censurer les partis politiques existant, ceux ne remplissant pas les conditions, les supprimer.

\section{CONCLUSION}

Eu gars à ceux qui précèdent, nous trouvons opportun de dire que le Congo est un jeune Etat « démocratique ». Il a encore besoin de beaucoup d'efforts pour arriver à maitriser le bon sens de la démocratie et ses principes. Afin, de l'adapter le mieux à sa nature juridique et aussi aux réalités africaines en général et congolaises en particulier. Sinon, au lieu que la démocratie soit pour le Congo (RDC) un moyen de développement, elle sera au contraire moyen de crises dans toutes ses dimensions, d'insécurités, violations des droits de l'homme et aussi de pauvreté.

Pour réussir la démocratie au Congo, marions-la avec le consensualisme du fait que le Congo est un Etat qui a une pluralité ethnique extraordinaire. Simple à comprendre est que lors qu'on a moins de partis, les différentes tribus peuvent se réunir dans un seul parti et développer au moyen du consensus un sentiment nationaliste ou unificateur. Par contre, lorsqu'on en plus des partis, chacun se rattachera à un parti dont leader est son frère ou sœur tribale. Ainsi, au lieu et place du sentiment nationaliste, c'est un sentiment séparatiste qui triomphera. Les répercutions et les effets ne seront que du sang dans la société congolaise.

Voilà en quels que mots, notre contribution à la bonne gouvernance de l'Etat du Congo et la mise en œuvre adéquate du principe de multipartisme démocratique.

\section{BIBLIOGRAPHIE}

\section{TEXTES LEGAUX}

1. Loi $n^{\circ} 11 / 002$ du 20 janvier 2011 portant révision de certains articles de la Constitution de la République Démocratique du Congo, Journal Officiel Numéro spécial 5 février 2011.

51 Veillez lire à ce sujet UROMME, « le Congo contemporain », p. 2. Article tiré de l'internet, http:// www.urome.be. 
2. La loi $n^{\circ} 07 / 008$ du 04 décembre 2007 portant statut de l'opposition politique

3. La loi $n^{\circ} 04 / 002 d u 15$ mars 2004 portant organisation et fonctionnement des partis politiques.

4. La Résolution du Dialogue inter-congolais $N^{\circ} \mathrm{DIC/CPJ/} 04$ du 18 avril 2002 relative à la libéralisation effective et totale de la vie politique et associative en République Démocratique du Congo.

\section{OUVRAGES}

5. A. DOWENS, An Economie Theory of Democracy, New York, Harper, 1957, P.24 (traduit par jean et Monica Charlot).

6. BANZA MALALE MAKUTA Gabriel, les aspects juridiques dans les enjeux des crises congolaises, des origines à nos jours (1860-2006).

7. Etienne ILUNGA KABULULU, Introduction génerale à l'étude du Droit, A l'intention des Cadres Administratifs de l'IG/PNC, RDC, JANVIER 2012.

8. Isidore NDAYWEL è NZIEM, La constitution de la IIIème République du Congo-zaïre, adoptée par la CNS? édition en 2 volumes, tome 1, L'Harmattan, 2002, p. 2-4.

9. Jacques Djoli Eseng'Ekeli, Droit constitutionnel, l'expérience congolaise (RDC), L'Harmattan, 2013, p. 81.

10. LAROUSSE, Dictionnaire de Français, ed. 2006.

11. MAURICE DUVERGER, Partis politiques, Paris, Edition A. Colin, 1968

12. Michael Walzer, pluralisme et démocratie, éditions esprit, 1997.

13. MULUMBATI NGASHA Adrien, introduction à la science politique, Lubumbashi, Edition africa, 1977.

14. NGOMA BINDA (P), La participation politique, IFEP, Kinshasa, 2005.

15. PAULA BECKER ET JEAN-AIME A., Qu'est-ce que la democratie?, Auteurs (texte original en allemand) : Paula Becker Traduction en français: Rabary-AndriamandayVoahanitriniaina Coordination : Jean-Aimé A. Raveloson Antananarivo, septembre 2008.

16. Roger-Schwartzenberg, sociologie politique, Paris, Ed. Montchrestien, 1974.

17. Serge GUINCHARD et Thierry DEBARD, Lexique des termes juridiques, Éditions Dalloz, Paris, 2018.

18. THIAM (D), Le Fédéralisme africain, Présence Africaine, Paris, 1972.

19. V. SAINT-JAMES, « Hiérarchie et conciliation des droits de l'homme ", J. ANDRIANTSIMBAZOVINA et alii (dir.), Dictionnaire des droits de l'homme, Paris, Puf, 2008, pp. 477-481

20. Yvan Dutil, Pour une meilleure démocratie Notions d'ingénierie électorale, Rapport présenté à la Commission des Institutions de l'Assemblée Nationale du Québec et au Comité directeur des États généraux sur la gouvernance démocratique, Novembre 2002.

\section{ARTICLES}

1. Babacar Gueye, «La démocratie en Afrique : succès et résistances », Pouvoirs/2 ( $\left.{ }^{\circ} 129\right)$, p. 5 - 26/

2. Biléou Sakpane-Gbati, «La democratie à l'africaine », in ethique publique, vol.13, $n^{\circ} 2$, 2011. https:// journals/openedition.org/ethiquepublique/679. 
3. Cheik FITA, " voici les 599 partis politiques et 77 regroupements pour les élections RDongo de $2018 », 15$ mai 2018. www.cheikfitanews.net/2018/05/voici-les-599-partis-politiques-et77-regroup ements-pour-les-elections. Consisté le 19 septembre $02 \mathrm{~h} 10$.

4. https://www.radiookapi.net/2018/03/20/actualite/politique/rdc. Consisté le 19 septembre 03h 19.

5. UROMME, « le Congo contemporain », p. 2. Article tiré de l'internet, http://www.urome.be.

7. YAV KATSHNG JOSEPH, RDC : «Nécessité d'un Profil Remodelé des Députés, Sénateurs, Gouverneurs, etc? », Le Contrôle Citoyen de la RDC, www.controlecitoyen.com,18 février 2010.

\section{NOTES DE COURS}

1. BANZA MALALE G., cours de Droit constitutionnel congolais, G2 Droit, UNILU, 2014-2015. Inédit

2. MUSAFIRI NALWANGO Paul, Cours de Droit constitutionnel : théorie générale de l'Etat, G1 Droit, UNILU, 2013-2014.

3. WHIKA TSHIBINDA BAUBOUIN, Cours de Droit structures et institution traditionnelles africains, G2 Droit, UNILU, 2014-2015. 